\title{
ANNOTATION
}

\section{The Place of Histochemistry Today}

Histochemistry is a young and progressive science which is at the same time of a respectable antiquity. It has its origins in the isolated investigations of a few biologists and botanists in the early Igth century and, especially clearly, in the carefully-planned work which the French botanist, Francois Vincent Raspail, performed during the 1820 .

Histochemistry may be defined as the chemistry of tissues and cytochemistry as the chemistry of cells, while the first term can naturally be used comprehensively to include the second. Broadly speaking, histochemistry covers a very wide field indeed, ranging from essentially biochemical methods of investigating tissue chemistry in vitro, through the in vivo or intra vitam techniques of the physiologist or cytologist, to the conventional methods of histologist or histopathologist, applied to tissue sections. This trichotomy, which began in the $1870 \mathrm{os}$, has remained with us since that day.

There has been considerable difference of opinion as to which of the various factions should use, unqualified, the two available titles and, in the United States, the term cytochemistry is often restricted to mean the use of non-histological techniques alone. Over here there is no agreement upon such usage but, nevertheless, throughout the world, the unqualified term histochemistry has come to be widely accepted as referring to the field in which the disciplines of histology and chemistry are blended. As such it deals with the localization in tissues and cells of chemically identifiable substances and reactivei groups, so that the concern of the applied histochemist, in whatever biological or pathologica $\mathrm{P}$ field his studies may lie, is to produce in a tissue $\vec{Z}$ section a localized reaction whose end result is visible under the microscope.

It was not until the early ig3os that the histochemistry of mounted tissues and cells, sometines called microscopical histochemistry, began overtake the biological branches of the science, with a host of new techniques which were produced to match the needs of histologists and cyto-0 logists to know something more of the structure whose morphology they had unravelled. At this time (1936) the first real textbook of histochemistry was published in France, written by Lison, whowas then an Assistant in the University of Brussels? This became, as Prenant has observed, ' le breviaireo de tous les histochimistes.' In succeeding years:histochemical methods, largely derived directly or indirectly from Lison, were used with in-0 creasing frequency by pathologists and histo logists. In the last few years great technicab advances have been made, and many of these have employed material or apparatus beyond the reach of all but specialists in small fields of res search. The majority, however, have fitted intos the normal pattern of practical microscopicato histochemistry.

There can be no clearer indication of presen interest in the histochemistry of tissue sections? than the existence of six current books or mono-0 
graphs dealing with this particular subject. These are, to name them in order of publication: Glick, ' Techniques of Histo- and Cytochemistry,' 1949; Panijel, 'Les Problemes de l'Histochimie et la Biologie Cellulaire,' I95 I ; Gomori, 'Microscopic Histochemistry,' 1952; Lison, 'Histochimie et Cytochimie Animales,' 1953; Pearse, 'Histochemistry, Theoretical and Applied,' 1953; and Danielli, ' Cytochemistry: A Critical Approach,' 1953. These books present between them the whole spectrum of the science, but the greatest emphasis is on the histochemistry of tissue sections.

There are today perhaps 150 histochemical techniques, 40 of them alone for localizing enzymes, and probably over a hundred of the larger total are technically within the compass of most workers familiar with the handling of cells and tissues. There is some difference of opinion, however, as to whether the interpretation of these methods is sufficiently easy to make their widespread use advisable or whether they should remain in the hands of a few specialists in histochemistry. In at least one of the six modern works which I have mentioned the author strongly suggests that the methods at present available are too imperfect, or their interpretation too difficult for their application to routine pathological problems to be of much benefit. Another author (Stowell, 1952), in a review of the uses of histochemical techniques, has stated that ' fundamental, critical research on new cytochemical technics will do more to advance our eventual understanding of normal tissues and neoplasia than the application of the relatively few and often none too satisfactory histochemical and cytochemical technics now available.'

With these views I strongly disagree, although I should certainly not wish to minimise the importance of the development of new methods of histochemical research by ' groups of collaborating investigators who have a thorough understanding of the fields of chemistry, physics and cytology.' In medicine the new and imperfect remedy does not await perfection by the research of groups of collaborating investigators in the pure sciences. It is applied forthwith to their patients by the practitioners of medicine and it is often by their observations and researches that real advancement in the use of the remedy, and in knowledge of its mechanism and meaning, is brought about. I believe very strongly, therefore, that the methods of modern histochemistry, despite their imperfections, should be applied by all practitioners in the biological, cytological and pathological sciences, to every variety of problem, whether morphological, physiological or pathological in nature.

All histologists are histochemists, whether they like it or not, for the information which they derive from conventional staining techniques is inevitably chemical or physical. Lack of proper knowledge of the mode of action of dyestuffs has not prevented their application to histology nor has it prevented the acquisition thereby of useful information. Largely as the result of such application of dyes a few of the conventional methods of dye staining have reached true histochemical status.

The various branches of histochemistry are not independent but interdependent, and none should be too proud to accept suggestion and directionk from the others. All are more or less subserviento to the chemistry, biochemistry or physics on which they depend. The importance of histo chemistry for the many cytologists, histologists and pathologists who wish to use its techniques lies in the fact that it offers an escape from static descriptive types of research into problems bearing on the behaviour of tissues and allows a dynamic and functional approach to normal cytology as well as to cellular pathology. The widespread use of histochemical techniques is limited, in my view, not so much by their imperfections or by difficulties in interpreting them, but by the time involved in mastering them and in maintaining in health condition the mass of solutions necessary for the various reactions. Where many techniques having equal or overlapping specificity ought to be employed there is too often time for one only. Applied histochemistry possesses the tools and the problems; what it needs is the time and the trouble. Alas, these two are more easy to describe than to achieve.

A. G. Everson Pearse

\section{REFERENCE}

STOWELL, R. E. (1952), Lab. Invest., 1, 210. 